\title{
Effects of Stationary Phase Cross-Linking and Ion-Exchange Capacity on the Retention of Carboxylic Acids in Ion-Exclusion Chromatography Using Sulfonated Resins
}

\author{
Kai Ling Ng, Greg W. Dicinoski, and Paul R. HADDAD ${ }^{\dagger}$ \\ Australian Centre for Research on Separation Science, School of Chemistry, University of Tasmania, \\ GPO Box 252-75, Hobart, Tasmania 7001, Australia
}

(Received April 5, 2001; Accepted June 6, 2001)

\section{Introduction}

Ion-exclusion chromatography (IEC) has been widely used in the separation of carboxylic acids, finding applications in the analysis of food and beverages, pharmaceuticals, biological samples, and acid rain samples. ${ }^{1}$ The retention mechanism of carboxylic acids in ion-exclusion chromatography has also been investigated in many studies, and the factors which affect retention can be divided into three categories: analyte effects, mobile phase effects, and stationary phase effects.

In IEC, the chromatographic system can be considered to consist of three phases: the mobile phase which comprises the eluent passing through the interstitial volume of the column, the stationary phase which comprises the occluded liquid trapped within the pores of the resin and the resin phase which is the solid resin network and functionalized groups. ${ }^{1}$ In this mode of chromatography, the analytes being separated have the same charge as the functional group on the resin; that is, anions are separated on cation-exchange columns and cations are separated on anion-exchange columns.

Modern IEC columns are usually packed with fully functionalized resins of polystyrene-divinylbenzene or polymethacrylate substrates. Most commercial ion-exclusion resins have approximately $8 \%$ cross-linking, 5 to $10 \mu \mathrm{m}$ particle size and $5 \mathrm{meq} / \mathrm{g}$ ion-exchange capacity. The functional groups for cation-exchange columns are usually sulfonate or carboxylate functional groups or a mixture of both. Previous studies investigating the effect of the ion-exchange capacity have covered only a small range of capacities ( 0 to $2 \mathrm{meq} / \mathrm{g}$ ) on highly cross-linked $(50 \%)$ polystyrene divinylbenzene resins., ${ }^{2,3}$ The retention of the carboxylic acids examined increased with increasing the ion-exchange capacity. This was opposite to the expected trend, as a higher concentration of functional groups on the resin should increase its electrostatic repulsion with the analyte, and hence decrease retention. Harlow and Morman have demonstrated that the degree of cross-linking of the resin has a profound effect on the retention of carboxylic acids. ${ }^{4}$ Weakly ionized species show more penetration into the occluded liquid phase on resins of low cross-linking than those of high cross-linking. They found that resins of lower crosslinking provided better resolution of highly ionized acids, such as $\mathrm{HCl}$, from organic acids.

\footnotetext{
$\doteqdot$ To whom correspondence should be addressed.
}

This paper presents a study of both these stationary phase effects by varying the ion-exchange capacity and degree of cross-linking of a polystyrene-divinylbenzene substrate over a wider range than has been investigated previously. Furthermore, unlike previous studies, which have only been performed on smaller columns, we packed the resins into $250 \times$ $8 \mathrm{~mm}$ chromatographic columns, which is typical of the size of standard IEC columns.

\section{Experimental}

\section{Instrumentation and reagents}

The ion chromatograph comprised a Dionex DX 500 system (GP40 pump, LC30 chromatography oven at $35^{\circ} \mathrm{C}, 50 \mu \mathrm{L}$ injector loop, and AD20 absorbance detector operated at 220 $\mathrm{nm}$ ). Chromatograms were recorded using Dionex Peaknet 5.1 software.

The sulfonated resins were packed into a $250 \times 8 \mathrm{~mm}$ chromatographic column using a Haskel (Burbank, CA, USA) pneumatic amplifier pump and water at 1600 psi as the packing solvent. The packed column was then equilibrated with $1 \mathrm{mM}$ sulfuric acid eluent at $1.00 \mathrm{~mL} / \mathrm{min}$ until a stable baseline was achieved. The retention volumes of 13 carboxylic acids (formic, acetic, propionic, butyric, valeric, malonic, succinic, glutaric, adipic, citric, benzoic, salicylic and phthalic acids) were measured on 6 resins of different ion-exchange capacities and degrees of cross-linking.

All chemicals and reagents, except those used in the sulfonation reactions, were of analytical grade and were used without further purification. The water used was purified using a Milli-Q water system (Millipore, MA, USA), followed by filtration through a $0.45 \mu \mathrm{m}$ filter.

\section{Sulfonation of polystyrene divinylbenzene resin}

The unfunctionalized polystyrene divinylbenzene substrate was obtained from Dionex Corporation (4\%, 8\% and 12\% cross-linking, average particle diameter of $8 \mu \mathrm{m}$ ), which is a microporous resin. Therefore, it has microscopic pores and a surface area of approximately 1 to $3 \mathrm{~m}^{2} / \mathrm{g}$. The fully functionalized resin has a density of approximately $1.3 \mathrm{~g} / \mathrm{mL}$. Two methods of sulfonation were used in order to obtain a greater range in the degree of sulfonation of the resins.

Sulfuric acid method. The first method was modified from the procedure used by Dumont and Fritz. ${ }^{5}$ The resin was mixed 
Table 1 Sulfonation conditions and ion-exchange capacity of sulfonated resins

\begin{tabular}{ccc}
\hline $\begin{array}{c}\text { \% Cross- } \\
\text { link }\end{array}$ & $\begin{array}{c}\text { Capacity } \\
\text { (mequiv/g) }\end{array}$ & Sulfonation condition \\
\hline 8 & 0.34 & Concentrated sulfuric acid, $240 \mathrm{~min}, 80^{\circ} \mathrm{C}$ \\
8 & 1.5 & $1 \%$ chlorosulfonic acid, $5 \mathrm{~min}, \mathrm{rm} \mathrm{temp}$ \\
8 & 2.8 & $1 \%$ chlorosulfonic acid, 30 min, rm temp \\
8 & 4.9 & $40 \%$ chlorosulfonic acid, $180 \mathrm{~min}, \mathrm{rm}$ temp \\
4 & 3.2 & $40 \%$ chlorosulfonic acid, $240 \mathrm{~min}, \mathrm{rm}$ temp \\
12 & 4.5 & $40 \%$ chlorosulfonic acid, $195 \mathrm{~min}, \mathrm{rm}$ temp \\
\hline
\end{tabular}

with $80 \mathrm{~mL}$ glacial acetic acid (AR, BDH Chemicals, Australia) for $30 \mathrm{~min}$ at the desired reaction temperature to form a slurry. Concentrated sulfuric acid (AR, Ajax Chemicals) was added to the reaction mixture while stirring. After the desired reaction time, the mixture was poured into ice to quench the sulfonation reaction and then filtered, washed with water, methanol and acetone, and dried in vacuo. The degree of sulfonation was controlled by the reaction temperature and time; the conditions used for $20 \mathrm{~g}$ of resin are given in Table 1.

In sulfonating the resin using the concentrated sulfuric acid method, the degree of sulfonation obtained was very low $(<1$ $\mathrm{meq} / \mathrm{g}$ ), even at higher reaction temperatures and time. Therefore, a second sulfonation method using chlorosulfonic acid was employed.

Chlorosulfonic acid method. The procedure was modified from a method used by Rabia et al. for the chlorosulfonation of styrene divinylbenzene co-polymers. ${ }^{6}$ Analogous to the above reaction using concentrated sulfuric acid, the resin was mixed with $240 \mathrm{~mL}$ of dry dichloromethane (HPLC grade, Ajax Chemicals) to form a slurry, to which was added various amounts of chlorosulfonic acid (99\%, Aldrich Chemicals, Australia) to yield a range of reaction mixtures of various chlorosulfonic acid concentrations. After the desired reaction time, the mixture was filtered and washed with dichloromethane and water. The sulfonated resin was then stirred in a $1 \mathrm{M}$ $\mathrm{NaOH}$ solution at room temperature to hydrolyze the sulfonyl chloride to sulfonic groups according to the following equation:

$$
\mathrm{ArSO}_{2} \mathrm{Cl}+\mathrm{NaOH} \longrightarrow \mathrm{ArSO}_{3} \mathrm{H}+\mathrm{NaCl}
$$

Finally, the resin was washed and filtered with water. The degree of sulfonation was controlled by varying the concentration of chlorosulfonic acid, the reaction time and the temperature. The reaction conditions for $12 \mathrm{~g}$ of resin are given in Table 1.

\section{Determination of ion-exchange capacity of resin}

The total ion-exchange capacity of each batch of sulfonated resin was determined by packing approximately $1.5 \mathrm{~g}$ (dry weight) of resin into a $250 \times 4 \mathrm{~mm}$ chromatographic column using water at $1500 \mathrm{psi}$. Using a chromatography pump, $50 \mathrm{~mL}$ of $1 \mathrm{M} \mathrm{HCl}$ was passed through the column at $1.00 \mathrm{~mL} / \mathrm{min}$ to ensure that the resin was in the $\mathrm{H}^{+}$-form. Excess $\mathrm{HCl}$ was removed by washing the column with water at $1.00 \mathrm{~mL} / \mathrm{min}$ until the effluent reached about $\mathrm{pH} 6$.

The $\mathrm{H}^{+}$sites on the resin were converted into $\mathrm{Na}^{+}$by passing $50.0 \mathrm{~mL}$ of $0.5 \mathrm{M} \mathrm{NaCl}$ at $1.00 \mathrm{~mL} / \mathrm{min}$ through the column, followed by a rinse with water. The effluent was collected in a volumetric flask, diluted to $100.0 \mathrm{~mL}$ and titrated with standardised $\mathrm{NaOH}$ to the Methyl Red end-point ( $\mathrm{pH} \mathrm{4.8-6).}$ The ion-exchange capacities were calculated as milliequivalents

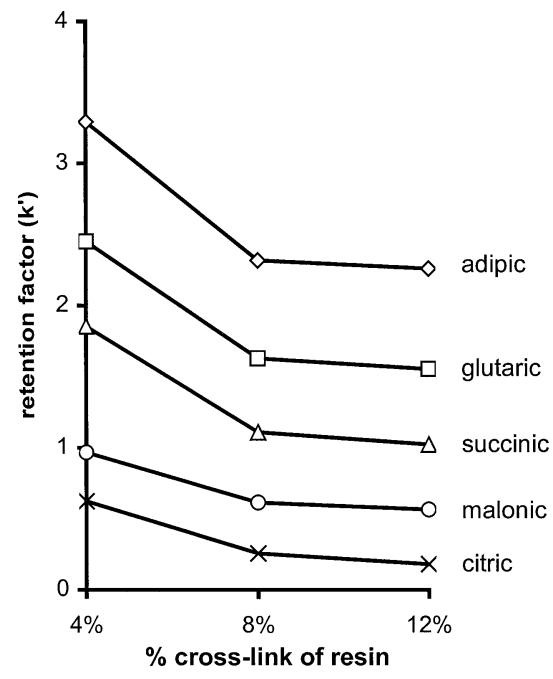

Fig. 1 Retention factors of dicarboxylic acids and citric acid on resins of different cross-linking.

$\mathrm{SO}_{3}{ }^{-}$per gram of resin, and are given in Table 1.

Collection of retention data and calculation of retention factors The void volume of each column $\left(V_{\mathrm{m}}\right)$ was determined by measuring the retention volume of a $0.1 \mathrm{mM}$ solution of nitric acid. The retention factors of the carboxylic acids were then calculated from its retention volume $\left(V_{R}\right)$ using

$$
k^{\prime}=\frac{V_{\mathrm{R}}-V_{\mathrm{m}}}{V_{\mathrm{m}}}
$$

When $k^{\prime}=0$, that is, elution is at the void volume, the analyte is completed excluded from the resin.

\section{Results and Discussion}

\section{Effect of the degree of cross-linking}

Figure 1 shows the retention factors of the dicarboxylic acids (malonic, succinic, glutaric and adipic acids) and citric acid, a tricarboxylic acid, on the three fully-sulfonated resins of different cross-linking. Whilst this comparison would preferably have been made using resins of the same capacity, the different structures of the three resins resulted in slightly different capacities for each resin. The higher cross-linked resins are more rigid with smaller pore sizes, and are hence less prone to swelling. Therefore, it is more difficult for the analyte to penetrate into the resin and the retention factors for those acids decreased with increasing cross-linking due to the increased repulsion of the analyte molecules by the surface sulfonate groups.

In the case of the monocarboxylic acids (Fig. 2), the retention factors decreased when the degree of cross-linking increased from $4 \%$ to $8 \%$ by a similar mechanism to that explained above in the case of dicarboxylic acids. However, an opposing trend was observed when the degree of cross-linking increased from $8 \%$ to $12 \%$. Previous studies have shown that hydrophobic adsorption exerts a significant influence on the retention mechanism of carboxylic acids in IEC. A higher cross-linked resin has a higher density, and hence a greater amount of resin is present per column volume than a resin of lower crosslinking. ${ }^{7}$ The increasing adsorption of the acid analyte onto the 


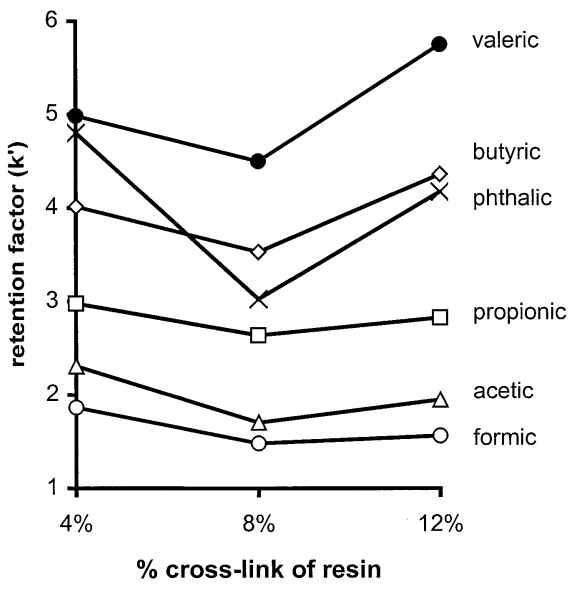

Fig. 2 Retention factors of monocarboxylic acids and phthalic acid on resins of different cross-linking.

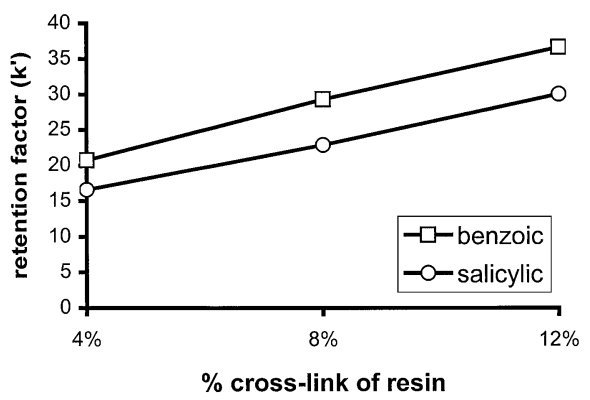

Fig. 3 Retention factors of benzoic and salicylic acids on resins of different cross-linking.

resin surface suggested that there was an increase in the hydrophobic area of the resin, which provided a competing mechanism for retention. The effect was more pronounced for those acids which were retained primarily by adsorption, especially the longer chain carboxylic acids (butyric and valeric acids) and aromatic species (such as phthalic acid). These acids showed a marked increase in retention on the $12 \%$ cross-linked resin over the $8 \%$ cross-linked resin.

The retention mechanism for benzoic and salicylic acids (Fig. 3) was dominated by hydrophobic adsorption due to the $\pi$ election interactions between the aromatic ring of the analyte and that of the polymer matrix. Higher cross-linked resins also have a higher concentration of divinylbenzene groups in the structure, which enhanced this effect. Therefore, the retention factors of these two aromatic acids increased with increasing degree of cross-linking of the resin from $4 \%$ to $12 \%$, as shown in Fig. 3.

\section{Effect of ion-exchange capacity}

Previous studies have shown that the retention of carboxylic acids increases with increasing ion-exchange capacity to a certain point, and then decreases with further increases in ionexchange capacity. ${ }^{2,3}$ In theory, the expected trend is for the retention to decrease as the number of functional groups on the resin increases, since the electrostatic repulsion between the ionized analytes and function groups should increase. Lee and Lord offered an explanation for the observed trend, whereby hydrogen bond donation from the non-ionized analyte species to the sulfonate groups on the resin contributed to the retention. ${ }^{2}$

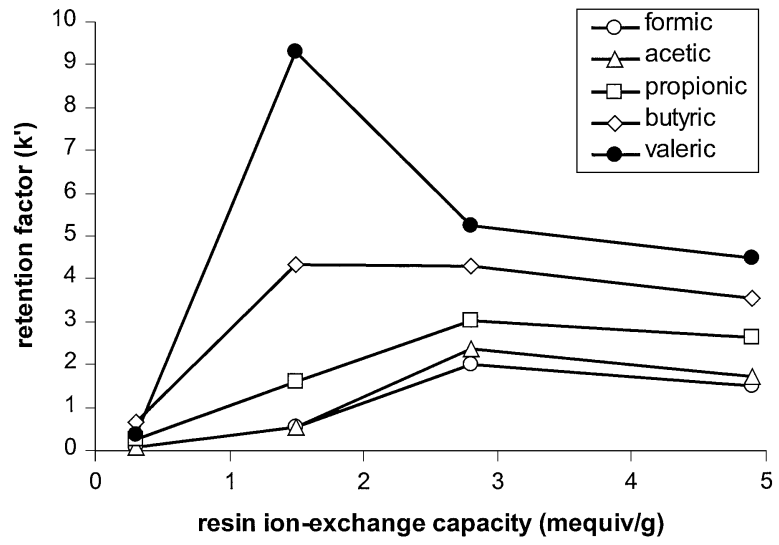

Fig. 4 Retention factors of monocarboxylic acids on $8 \%$ crosslinked resin of varying ion-exchange capacity.

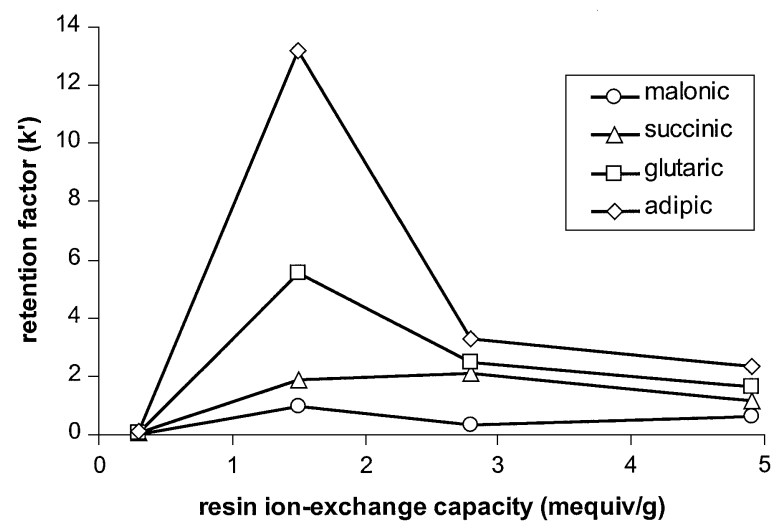

Fig. 5 Retention factors of dicarboxylic acids on $8 \%$ cross-linked resin of varying ion-exchange capacity.

Therefore, the retention of weakly ionized species increased with increasing concentration of sulfonate functional groups.

Figure 4 shows the retention factors of five monocarboxylic acids on resins of varying ion-exchange capacity. A similar trend to the previous studies was observed with the retention factors increasing when the ion-exchange capacity increased from 0.5 to 1.5 mequiv/g for all acids, while the retention factors of formic, acetic and propionic acids increased even up to an ion-exchange capacity of 2.8 mequiv/g. For analytes in which adsorption was the primary retention mechanism (e.g. butyric and valeric acids) a more profound effect was observed, leading to a greater increase in the retention factor. As the ionexchange capacity increased even more, the retention mechanism became dominated by electrostatic repulsion, and hence the retention decreased with increasing concentration of the functional groups for all acid analytes.

A similar trend was observed for the dicarboxylic acids (malonic, succinic, glutaric and adipic acids), as shown in Fig. 5. A surprising result was also observed for the 1.5 mequiv/g resin, with the retention factor of adipic and glutaric acids being higher than their corresponding monovalent acids, valeric and butyric acids. We suggest that the divalent acids are more strongly retained due to the possibility of forming twice as many hydrogen bonds with the sulfonate functional groups on the resin. 


\section{Conclusion}

The properties of the stationary phase substrate can have a profound effect on the retention mechanism of carboxylic acids in ion-exclusion chromatography, which leads to the possibility of optimizing the nature of the stationary phase in order to achieve the separation of some carboxylic acids. In this study, we investigated the effects of varying the ion-exchange capacity and degree of cross-linking of the polystryrene-divinylbenzene substrate over a wider range than has been studied previously. Although commercially available ion-exclusion chromatography resins are usually fully sulfonated $8 \%$ polystyrene divinylbenzene resins, a lower cross-linked resin, or a resin of lower ion-exchange capacity, might be desirable for the separation of highly ionized acids.

\section{Acknowledgements}

The authors would like to thank Dionex Corporation for supplying the unfunctionalized AS1 resin and Andy Woodruff (Dionex Corporation, USA) for his technical expertise in the sulfonation procedures.

\section{References}

1. P. R. Haddad and P. E. Jackson, "Ion Chromatography-Principles and Applications", 1991, Elsevier, Amsterdam.

2. D. P. Lee and A. D. Lord, $L C-G C, 1987,5(3), 261$.

3. C. W. Klampfl, W. Buchberger, G. Rieder, and G. K. Bonn, J. Chromatogr. A, 1997, 770, 23.

4. G. A. Harlow and D. H. Morman, Anal. Chem., 1964, 36, 2438.

5. P. J. Dumont and J. S. Fritz, J. Chromatogr. A, 1995, 691, 123.

6. I. Rabia, J. Zerouk, M. Kerkouche, and M. Belkhodja, React. Function. Polym., 1996, 28, 279.

7. Personal communication, R. L. Albright, 1995, Albright Consulting. 